\title{
Influence of the rotor eccentricity on the torque of a cage induction machine
}

\author{
Bishal Silwal $^{1}$, Pajyo Rasilo ${ }^{1,2}$, Anouar Belahcen ${ }^{1}$, Antero arkkio ${ }^{1}$ \\ ${ }^{1}$ Department of Electrical Engineering and Automation, Aalto University \\ P.O. Box 13000, FI-00076 AALTO, Finland \\ ${ }^{2}$ Department of Electrical Engineering, Tampere University of Technology \\ P.O. Box 692, FI-33101, Tampere, Finland \\ email: bishal.silwal@aalto.fi
}

(Received: 12.08.2016, revised: 08.02.2017)

\begin{abstract}
The non-uniform air gap in an electrical machine caused by rotor eccentricity creates an asymmetrical flux-density distribution in the air gap. This can affect the nominal torque produced by the machine. Eccentricity also produces forces that act on the rotor which may also have an effect on the torque. Thus, it is important to know how the torque of the machine behaves. In this paper, the torque of a cage induction machine is studied when the machine has dynamic eccentricity. The study is performed using the finite element method and a magnetic vector potential formulation. The torque is calculated by the method of energy balance. The harmonic components of the torque are also analyzed. The results show that the machine under eccentricity does not exhibit the same torque as a normal healthy machine. The harmonic components around the first principal slot harmonic is most affected.
\end{abstract}

Key words: cage induction machine, dynamic eccentricity, electromagnetic torque, energy balance, finite element method, harmonics

\section{Introduction}

The rotor of a radial flux electrical machine is ideally supposed to be concentric with the stator which may not be always true. The rotor may be shifted from the center point or it may also be whirling around the center point in a circular path at a certain frequency and with a small radius, as shown in Fig. 1. Conventionally, there are two types of eccentricity. Static eccentricity corresponds to the situation where the rotor is just shifted from the center point but does not make any whirling motion. In dynamic eccentricity, the shifted rotor also makes a whirling motion, i.e. the center point of the rotor rotates around the center point of the stator at a certain frequency and with a certain radius. Rigorous studies have been already done in the past to study the eccentricity in a cage induction machine, and the forces caused by it [1-3]. However, most of the literature focuses on an eccentric cage induction machine and the 
forces and vibrations related to it. The unbalanced magnetic pull due to rotor eccentricity has also been a topic of investigation in the past [4-6]. The analytical models have been validated by experimental investigations.

However, very few publications regarding the study of the behavior of the torque in the case of an eccentric machine were found. The electromagnetic torque of an electrical machine is very important since it is used to convert the electrical power into mechanical power to the shaft of the machine. The torque in eccentric switched reluctance machines was studied in [7]. [8] presents a study on a similar problem in the same type of machine. A brief discussion about the difference in the torque behavior of a healthy machine and an eccentric machine can be found in [9]. An analytical study of a similar problem for an induction machine has been done in [10]. Some studies have also been done for the induction machine under mixed eccentricity, which is a case when the machine has both static and dynamic eccentricities simultaneously. For instance, [11] presents the analysis of stator current under mixed eccentricity and also explains the influence of the load variations on different harmonic components induced in the current spectrum due to the eccentricity. [12] employs the stator current spectrum for the diagnosis of the mixed rotor eccentricity and also presents the effect of mixed eccentricity on the time variation of the torque. [13] briefly compares the peak value of the instantneous torque in the torque of an induction motor for a healthy case and cases with static, dynamic and mixed eccentricities but the results presented are not comprehensive enough to have a concrete conclusion. In this paper, we comprehensively investigate the effect of the dynamic rotor eccentricity on the electromagnetic torque of a cage induction machine numerically, using finite element analysis. The analysis will be based on the average torque, time variation of the torque and the harmonic components in the torque waveform.

The torque calculation using the finite element method (FEM) is very common, especially while designing an electrical machine and a handful of publications can be found about computing the torque. There are methods based on the magnetic coenergy [14] and stored energy [15] but typically the Maxwell stress tensor method [16] and Coulomb's method based on the principle of virtual work [17] are used for the numerical computation of torque. However, these methods do not ensure an accurate torque estimate in the case of an eccentric machine. When the torque is integrated over the elements in the air gap of the machine, the radius of integration is important. Generally, the radius of integration is taken by considering the geometrical center point of the stator as the center, which, in the case of an eccentric machine, is not true. This has been explained figuratively in [18].

In this paper, the method of energy balance has been used to compute the torque. This method is found to be accurate and insensitive to the type of mesh used in the air gap of the machine. Recently, a study compared the energy balance method and Coulomb's virtual work method based on the type of discretization used in the air gap [19]. The average torque calculated from Coulomb's virtual work method is dependent on the density of the mesh and the shape of the elements. It has also been seen to be affected by the uniformity of the mesh. In the case of dynamic eccentricity, the finite element modeling of whirling makes the nonuniformity of the airgap inevitable in the air gap. Therefore, the use of energy balance method to compute the torque ensures a better accuracy. 


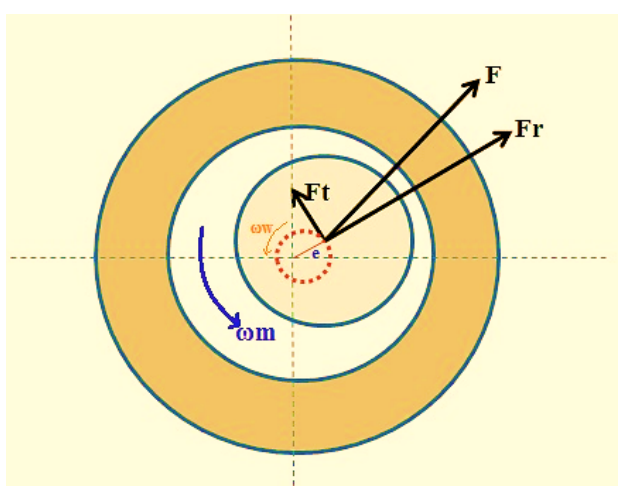

Fig. 1. Forces due to eccentricity. $F_{r}$ and $F_{t}$ are the radial and the tangential components of the force, respectively

\section{Method of analysis}

\subsection{Time-stepping finite element method}

The magnetic field in the cross section of the machine is calculated by using two-dimensional finite element analysis. The time-stepping method is based on the $\boldsymbol{A}-\Phi$ formulation where $\boldsymbol{A}$ is the magnetic vector potential and $\Phi$ is the reduced electric scalar potential. The Maxwell field equations in the quasi-static state together with the constitutive material equations lead to the following equation to be solved in the cross-sectional geometry of the machine.

$$
\nabla \times(v \nabla \times A)=-\sigma\left(\frac{\delta A}{\delta t}-\nabla \Phi\right),
$$

where: $v$ is the reluctivity and $\sigma$ is the conductivity of the material. One period of the fundamental frequency is divided into 800 time steps and second order triangular elements are used for the study. The whole mesh has 4290 elements and 8677 nodes. Part of the mesh showing the air gap region is shown in Fig. 2. The magnetic field in the machine is assumed to be twodimensional. The three dimensional end winding fields are modeled approximately by adding the end-winding impedances to the circuit equations of the windings. Trapezoidal rule is used for time integrations. The nonlinearity of the materials is taken into account by using a single valued magnetization curve. The details of the method have been presented in [20].

The movement of the rotor is taken into consideration by the moving-band technique. The center position of the rotor is forced to move along a circular path at a constant speed to model the dynamic eccentricity. In addition, the rotor was rotated at its mechanical angular speed around its geometrical center.

An analytical derivation predicts that the eccentric rotor motion induces two eccentricity harmonics in the air-gap flux density [1,21]. When expressed in the stator frame of reference, they are: 


$$
\begin{aligned}
& B_{p+1}=\hat{B}_{p+1} \cos \left[(p+1) \phi-\left(\omega_{s}+\omega_{w}\right) t+\alpha_{p+1}\right\rfloor, \\
& B_{p-1}=\hat{B}_{p-1} \cos \left[(p-1) \phi-\left(\omega_{s}-\omega_{w}\right) t+\alpha_{p-1}\right],
\end{aligned}
$$

where: $\hat{B}$ is the peak value of flux density, $p$ is the number of pole pairs, $\omega_{S}$ is the supply frequency and $\omega_{w}$ is the whirling frequency. These eccentricity harmonics together with the fundamental flux density create an asymmetrical flux-density distribution which causes the forces that act on the rotor.

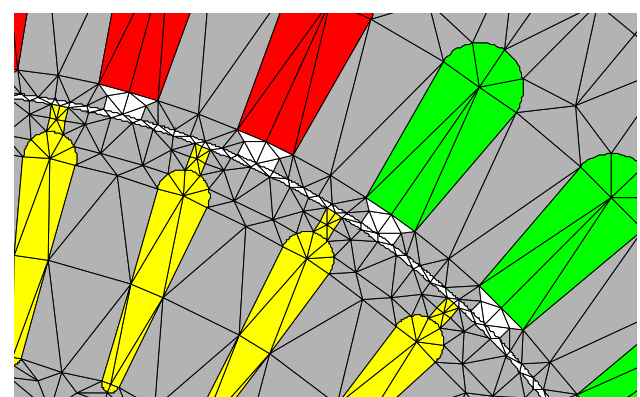

Fig. 2. Mesh used in the simulation

\subsection{Energy balance method}

The primary focus of this study is the torque and its harmonic components. In this paper, the torque is calculated by the method based on the energy balance of the machine. In this method, if the angular speed of the rotor is constant, the power balance of the machine can be integrated for a certain period of time to obtain the average torque over the time interval. The expression for the torque computed from this method is given by:

$$
T^{a}=\frac{P_{\text {in }}^{a}-P_{\text {loss }}^{a}-\frac{\Delta W_{t}}{\Delta t}-P_{\text {whirl }}^{a}}{\omega_{m}^{a}},
$$

where: $P_{\text {in }}$ is the input power, $P_{\text {loss }}$ is the electromagnetic loss, $W_{f}$ is the energy of the electromagnetic field, $P_{\text {whirl }}$ is the power produced by the tangential component of the force and the velocity vector of whirling, $\omega_{m}$ is the mechanical angular speed of the rotor and the superscript ${ }^{a}$ denotes the average value. The whirling power, $P_{\text {whirl }}$, gets either added to or subtracted from the system depending on the direction of the force. This power becomes significant under eccentricity operation and therefore it has to be taken into consideration when the power balance is used to calculate the force. But since the torque corresponding to this power is very small, we can neglect it in the present study.

In the present study, the stator winding has been modeled as a filamentary winding without eddy currents. The input power to the machine is calculated using the currents and the flux linkages of the stator phases 


$$
P_{\text {in }}^{\mathrm{a}}=\frac{\Delta W_{\mathrm{in}}}{\Delta t}=\frac{1}{\Delta t} \sum_{k=1}^{m} \int_{\Psi_{k i}}^{\Psi_{k f}} i_{k} \mathrm{~d} \Psi_{k},
$$

where: $i_{k}$ is the current of phase $k, \psi_{k i}$ and $\psi_{k f}$ are the flux linkages of phase $k$ at the beginning and end of the period $\Delta t$, respectively. $m$ is the number of phases.

$P_{\text {loss }}$ is obtained as:

$$
\begin{aligned}
P_{\text {loss }}^{\mathrm{a}} & =\frac{\Delta W_{\text {loss }}}{\Delta t}=\frac{1}{\Delta t} \int_{V}^{t_{0}} \int_{t_{0}}^{+\Delta t}\left(-E \cdot \frac{\partial \mathbf{A}}{\partial t}\right) \mathrm{d} t \mathrm{~d} V, \\
& =\frac{1}{\Delta t} \int_{V}^{t_{0}} \int_{t_{0}}^{+\Delta t} \sigma\left(\frac{\partial \mathbf{A}}{\partial t}-\frac{u}{l} \mathbf{e}\right) \cdot \frac{\partial \mathbf{A}}{\partial t} \mathrm{~d} t \mathrm{~d} V
\end{aligned}
$$

where: $V$ is the volume of the solution region, $E$ is the electric field strength, $u$ is the electric scalar potential and $l$ is the length of the machine. In the simulations, the core materials have been treated as non-conducting, and thus the core losses are not included in the present study.

The change in the magnetic field energy $W_{f}$ over the period of time is calculated from the magnetization curves of the materials and flux densities $B_{0}$ and $B_{t}$ at the beginning and the end of the period.

$$
\Delta W_{f}=\int_{V}^{B_{t}} \int_{B_{0}}^{\boldsymbol{H}} \cdot \mathrm{d} \boldsymbol{B} \mathrm{d} V .
$$

If one is interested in the instantaneous torque, $\Delta t$ in Eq. (4) can be equal to the length of one time step. For an average torque in the steady state of the machine, the time interval $\Delta t$ should be chosen to be an integer multiple of the fundamental period of the machine. The time derivatives are approximated by first order difference ratios and the time integrals in Eqs (4-7) are summed up time-step by time-step. Over one time step, the input energy and the energy consumed by the resistive loss in the rotor cage are respectively,

$$
\begin{gathered}
\Delta W_{\text {in }}=\sum_{n=1}^{m}\left[\beta i_{n, k+1}+(1-\beta) i_{n, k}\right]\left(\Psi_{n, k+1} \Psi_{n, k}\right), \\
\Delta W_{r t}=\int_{V} \sigma\left\{\left[\begin{array}{c}
\frac{A_{k+1}-A_{k}}{\Delta t} \\
\frac{-1}{l}\left[\beta u_{k+1}+(1-\beta) u_{k}\right] \boldsymbol{e}_{z}
\end{array}\right] \cdot\left(\boldsymbol{A}_{k+1}-\boldsymbol{A}_{k}\right)\right\} \mathrm{d} V .
\end{gathered}
$$

where $\beta=0.5$ for the trapezoidal rule. 


\section{Results and discussion}

A 4-pole $15 \mathrm{~kW}$ cage induction machine, the parameters of which are given in Table 1, is used as a test machine in this study. The method of analysis for the calculation of the eccentricity forces has been validated earlier by modeling and measuring this machine [1]. A part of the geometrical cross-section of the machine along with the calculated magnetic flux density distribution is shown in Fig. 3. The eccentric rotor, displaced from its center position can be clearly seen from the figure. The force produced by the eccentricity is directed towards the shortest air gap. The magnetic flux density is also higher in that region, as can be seen from the figure.

Table 1. Parameters of the test machine

\begin{tabular}{l|c}
\hline Parameter name & Value \\
\hline Number of poles & 4 \\
\hline Connection & Delta \\
\hline Rated voltage [V] & 380 \\
\hline Supply frequency & 50 \\
\hline Rated current [A] & 31 \\
\hline Rated power [kW] & 15 \\
\hline
\end{tabular}

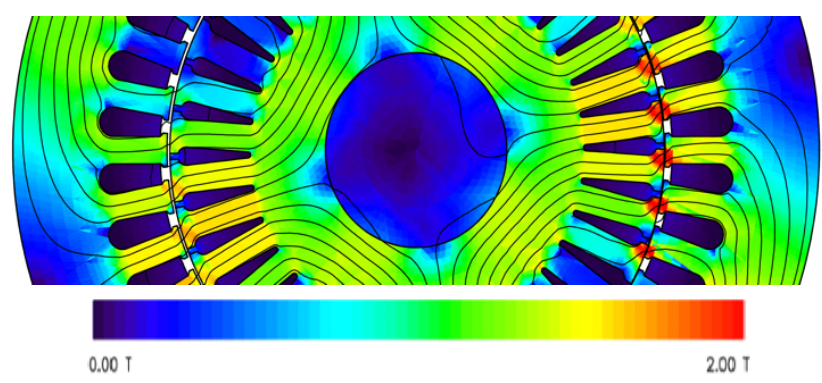

Fig. 3. Flux density distribution in the cross-section of an eccentric machine

Many simulations were performed by varying the whirling radius in each simulation. Initially the torque computed from the energy balance method is compared with that computed from the Coulomb's method for different types of mesh used in the air gap. For this purpose, the air gap of the machine was discretized with single, double and triple layers of elements. For a single layer mesh, both the torque computation and the movement of the rotor is done using the same band of elements. But for the double and triple layer mesh, the band of elements used for the torque computation and the movement of the rotor may be the same or different. In this way, altogether 14 different combinations of the number of layers, and the bands used for the torque computation and the rotor movement were used for comparison and the result is shown in Fig. 4. The eccentricity in this case was 33\% and the machine was rotated at its rated speed. The average torque computed from Coulomb's method varies with 
the variation in the type of mesh. However, the energy balance method gives an average torque almost independent from such variations. Therefore, the energy balance method seems more accurate and has been further used to compute the torque in this paper.

Fig. 5 shows the average torque of the machine as a function of the whirling radius. All the simulations were performed at the rated slip of the machine, which is $3.2 \%$. It is clear from the results that an electrical machine under eccentricity does not exhibit the same torque as a healthy machine. The results show an increase in the torque of the machine with increasing eccentricity. The results at $0 \%$ relative eccentricity refer to a concentric healthy machine.

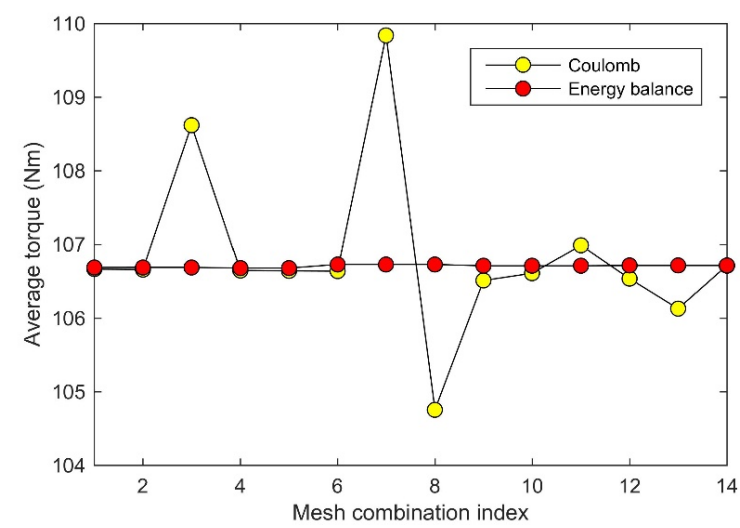

Fig. 4. Average torque calculated with different types of mesh in the air gap. Mesh index notation: $X Y Z$, $X=$ number of layers, $Y=$ movement layer, and $Z=$ torque computation layer; $1=111,2=211,3=212$, $4=221,5=222,6=311,7=312,8=313,9=321,10=322,11=323,12=331,13=332$, and $14=333$

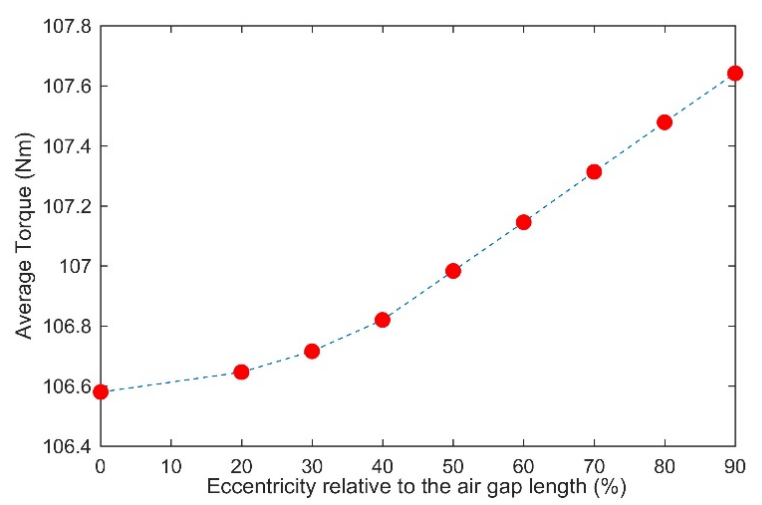

Fig. 5. Average torque as a function of the whirling radius

In addition to the average torque, the influence of the eccentricity on the harmonic contents in the torque is analyzed. The time variation of the torque is shown in Fig. 6. Since the energy balance method gives only the average torque over an interval of time, the time variation was obtained by using a period of integration equal to the time step size used in the simulations. 
The time-axis of the figure has been zoomed-in to clearly see the time variation of the torque at different eccentricity levels. An increased distortion in the torque waveform with the increase in relative eccentricity can be witnessed from the figure.

The harmonic components of the torque are computed using fast Fourier transform (FFT). The harmonic distortions of the torque waveform for different relative eccentricities are shown in Table 2. Fig. 7 shows the frequency spectrum of the torque for a healthy machine and Fig. 8 shows the frequency spectrums of the torque for the machine with dynamic eccentricity. The figures show the frequency range of $200-2000 \mathrm{~Hz}$ because the harmonic components having considerably larger amplitude, other than the fundamental component are seen to occur in this frequency range. For a healthy machine, some of the largest torque components are found to be at $300 \mathrm{~Hz}$ and $1700 \mathrm{~Hz}$. In the case of dynamic eccentricity, the $870 \mathrm{~Hz}$ component becomes significant and the behavior of this component is quite interesting as the results show a drastic increase in the amplitude of this component with the increase in the eccentricity. The $600 \mathrm{~Hz}$ component also shows similar behavior with the increase in eccentricity.

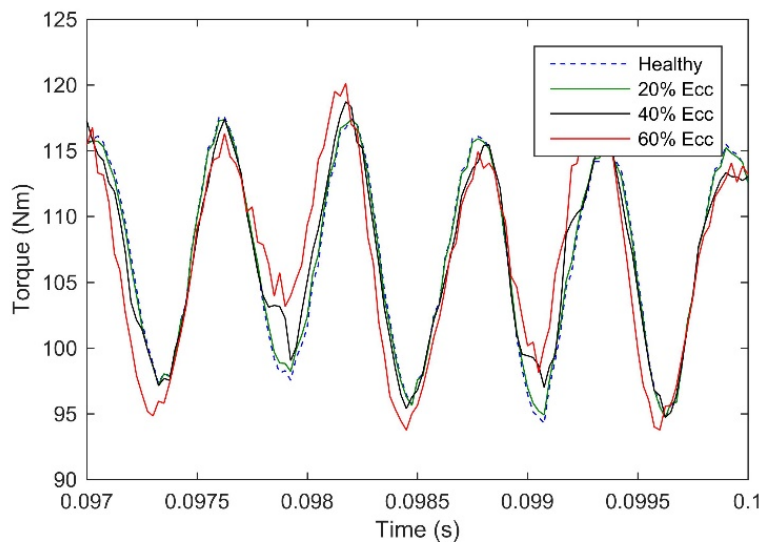

Fig. 6. Time variation of the torque at different eccentricity levels

Table 2. Harmonic distortion in torque

\begin{tabular}{c|c}
\hline Relative eccentricity [\%] & Distortion [\%] \\
\hline 0 & 38.05 \\
\hline 20 & 38.22 \\
\hline 30 & 38.59 \\
\hline 40 & 39.77 \\
\hline 50 & 42.19 \\
\hline 60 & 46.01 \\
\hline 70 & 49.87 \\
\hline 80 & 54.36 \\
\hline 90 & 61.26
\end{tabular}


Among other larger harmonics, the $300 \mathrm{~Hz}$ component also shows a slight increase in the amplitude, but it is very small compared to the increase in the $870 \mathrm{~Hz}$ component. The higher order harmonic around $1700 \mathrm{~Hz}$ has a slightly reduced amplitude with increasing eccentricity.

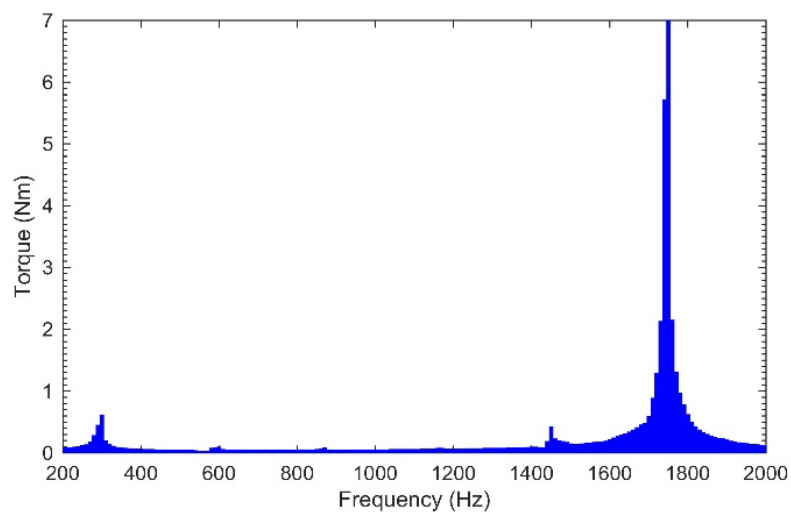

Fig. 7. Frequency spectrum of the torque for a healthy machine
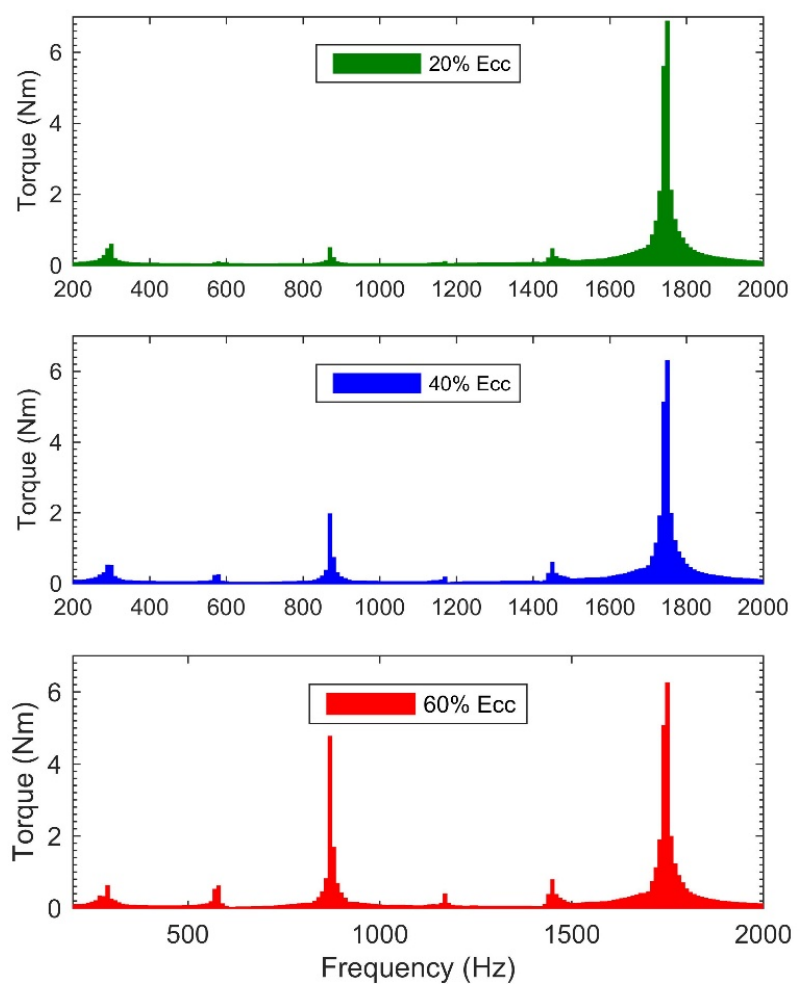

Fig. 8. Frequency spectrums of the torque in case of dynamic eccentricity 
The origin of the harmonic components of the torque can be found in the harmonics of the stator current. Therefore, to investigate further, the harmonic components in the stator current are studied. Fig. 9 shows the waveform of the line currents in the stator windings, simulated at the rated load, for a healthy machine. The frequency spectrum of the line current $i_{a}$ is shown in Fig. 10. The frequency of the principal slot harmonic $(\mathrm{PSH})$ for the type of machine under study can be estimated from

$$
P H S=f_{s}+k \times Q_{r} \times(1-s) \times \frac{f_{S}}{p},
$$

where: $f_{S}$ is the supply frequency, $Q_{r}$ is the number of rotor slots, $s$ is the slip, $p$ is the number of pole pairs and $k=1,2,3$, etc. At the rated slip, the $P S H$ should occur around $872.8 \mathrm{~Hz}$. For the $P S H$ to be seen in the current spectrum, the pole pair number $Q_{r} \pm n p$, where $n$ is the harmonic order, should be equal to the pole pair number of the space harmonics produced by the stator winding [22]. The machine under study is a cage induction machine with the number of rotor slots $\left(Q_{r}\right)=34$. In a healthy machine, this number of rotor slots does not give a PSH and therefore it does not appear in the current spectrum shown in Fig. 10. However, the rotor slot harmonics exist and can be seen around $1695.6 \mathrm{~Hz}$.

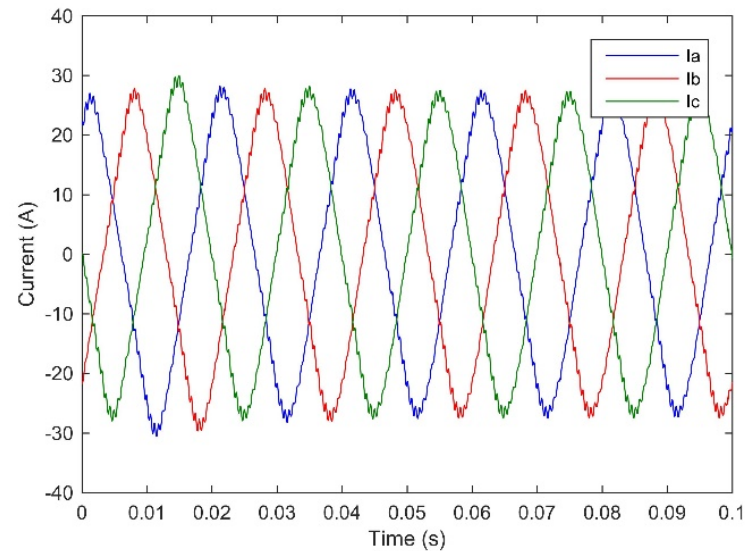

Fig. 9. Waveform for three-phase line currents, for a healthy machine

When the machine is under dynamic eccentricity, the harmonics corresponding to $n f_{s} \pm 2 f_{w}$ appear in the spectrum, where $n$ is the harmonic order in the supply and $f_{w}$ is the whirling frequency. Although, the $P S H$ does not appear in the healthy machine, $P S H \pm 2 f_{w}$ components exist in the eccentric case, and it can be seen from Fig. 11 that these components show a significant increase in their amplitude with the increase in the eccentricity. This explains the drastic increase in the torque component around $870 \mathrm{~Hz}$. However, the eccentricity harmonics corresponding to the second order PSH do not show much change in their amplitudes, the result of which is also seen in the spectrum of the torque shown in Fig. 8. Nonetheless, some other harmonics around $600 \mathrm{~Hz}$ are also slightly affected by the eccentricity, which is in turn also seen in the torque spectrum. 


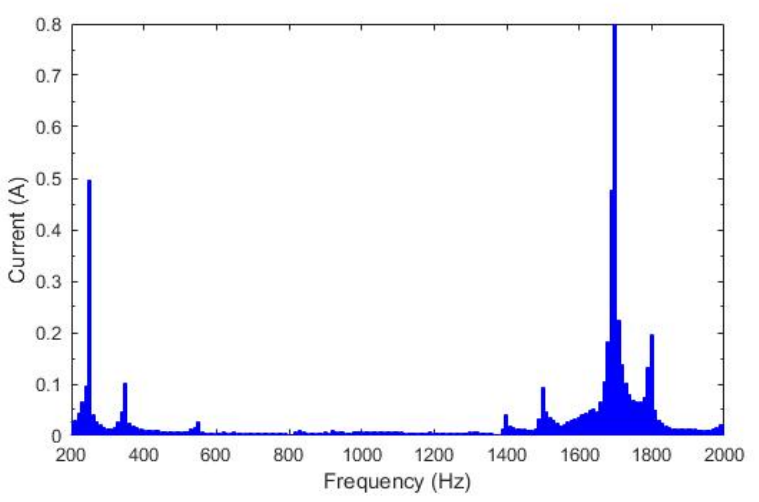

Fig. 10. Frequency spectrum of the stator line current $i_{a}$ healthy machine

For additional assessment of the torque in the machine with dynamic eccentricity, the machine was simulated at a quarter load, half load and full load. Table 3 shows the average torques computed for each case. Fig. 12 shows the differences between the torques of an eccentric motor and a non-eccentric motor for each case of loading, with different values of eccentricity. The results show how the difference between the torques of a healthy machine and an eccentric machine increases with eccentricity in different loading conditions.
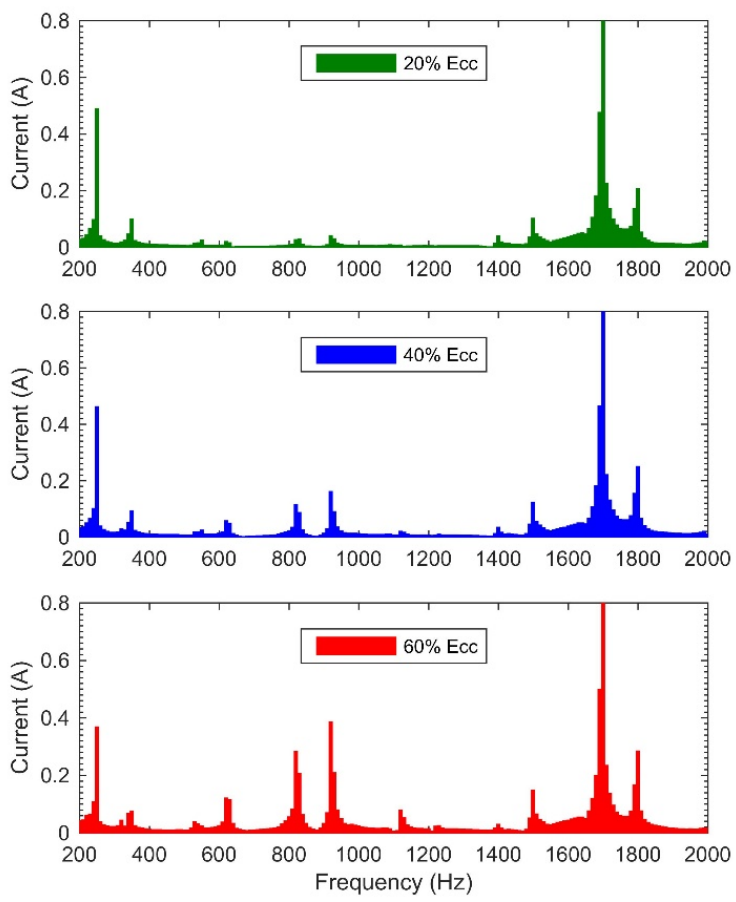

Fig. 11. Waveform for three-phase current in the stator winding, with dynamic eccentricity 
Table 3. Average torque values for different loading cases with different values of eccentricity [Nm]

\begin{tabular}{c|c|c|c}
\hline Eccentricity [\%] & Quarter load & Half load & Full load \\
\hline 0 & 28.6606 & 56.1111 & 106.5773 \\
\hline 20 & 28.7081 & 56.1860 & 106.6427 \\
\hline 40 & 28.8341 & 56.4089 & 106.8095 \\
\hline 60 & 29.0121 & 56.7683 & 107.1187 \\
\hline 80 & 29.1843 & 57.2064 & 107.4045 \\
\hline
\end{tabular}

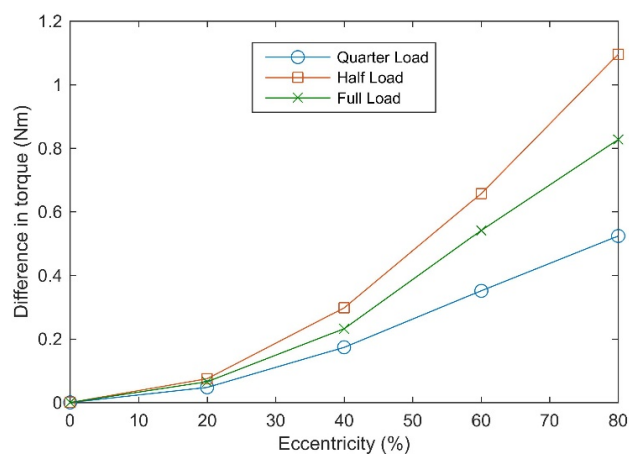

Fig. 12. Difference between the torque of an eccentric motor and a non-eccentric motor with different cases of loading

\section{Comparison with measurements}

A comparison between the simulated and the measured torque spectrums has been done for the case of static eccentricity. The measurement system consists of a $15 \mathrm{~kW}$ cage induction machine as a test machine, suspended with four horizontal arms connected to the frame of the machine in both the drive and the non-drive ends. The machine is equipped with active magnetic bearings used to create eccentricity and the whirling motion of the rotor. Four piezoelectric force sensors are placed vertically beneath the horizontal arms, which measure the vertical forces. The force sensors measure the dynamic force components only. The torque is extracted from the force. The harmonic components of the torque are obtained by performing an FFT for the torque signal. More details about the measurement set-up and the measurement process can be found in [23].

Fig. 13 shows the measured spectrum of the torque together with those obtained from the simulations. A locked rotor condition with a sinusoidal supply of $25 \mathrm{~V}$ was used both in the measurement and simulations. Fig. 13(a) shows a comparison for a healthy case and Fig. 13(b) shows that for $22 \%$ and $33 \%$ static eccentricity. The additional harmonics introduced in the eccentric case can be clearly observed from the figure. However, the simulation does not seem to yield similar results. This could possibly be for the reason that at $25 \mathrm{~V}$, the machine is still linear in the simulations. Using higher voltage in the simulation yields the harmonics which are not visible for $25 \mathrm{~V}$. This has also been explained in [23]. 


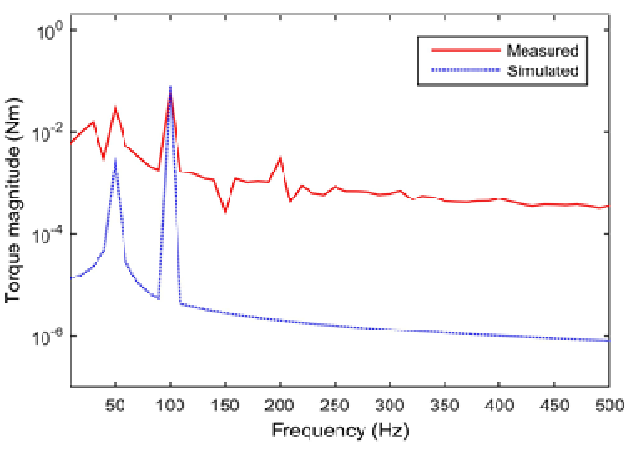

(a)

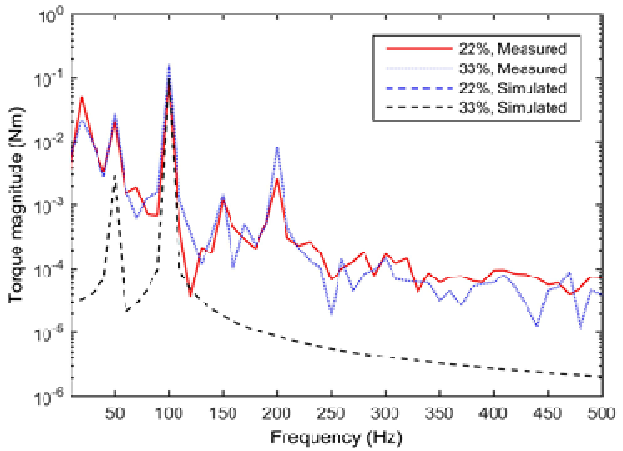

(b)

Fig. 13. Comparison between simulation and measurement: (a) healthy case; (b) static eccentricity case

\section{Conclusion}

The torque of a cage induction machine with a dynamic eccentric rotor has been studied. The finite element analysis is used for the study. Initial comparison between the Coulomb's virtual work method and the energy balance method showed the energy balance method to be more accurate, especially when the meshing in the air gap is considered. The investigation of torque revealed that the torque of an eccentric motor is relatively larger compared to the normal healthy machine. The torque harmonics were also investigated and the harmonic component with frequency close to the $P S H$ was found to have significant increase with the increasing eccentricity. Other larger harmonics were slightly affected. The additional torque might play an important role to induce torsional vibrations in the case when two machines are coupled and one of them becomes eccentric due to some practical problem, for instance, bearing breakdown or misalignment. The knowledge of the behavior of the harmonic components can also be applied for the detection of faults related to the eccentricity. To validate the simulations, the torque spectrums of the machine with static eccentricity obtained from both measurements and simulations were compared. The additional harmonics due to the static eccentricity were observed in the measurements.

\section{References}

[1] Arkkio A., Antila M., Pokki K., Simon A., Lantto E., Electromagnetic force on a whirling cage rotor, IEE Proceedings on Electric Power Applications, vol. 147, n. 5, pp. 353-360 (2000).

[2] Tenhunen A., Benedetti T., Holopainen T.P., Arkkio A., Electromagnetic forces in cage induction motors with rotor eccentricity, International Electric Machines and Drives Conference 2003, vol. 3, pp. 1616-1622 (2003).

[3] Arkkio A., Nepal B.R., Sinervo A., Electromechanical interaction in a synchronous reluctance machine, SPEEDAM 2010, pp. 501-506 (2010).

[4] Dorrell D.G., Smith A.C., Calculation of UMP in induction motors with series or parallel winding connections, IEEE Transactions on Energy Conversion, vol. 9, no. 2, pp. 304-310 (1994). 
[5] Smith A.C., Dorrell D.G., Calculation and measurement of unbalanced magnetic pull in cage induction motors with eccentric rotors, I. Analytical model, IEE Proceedings on Electric Power Applications, vol. 143, no. 3, pp.193-201 (1996).

[6] Dorrell D.G., Smith A.C., Calculation and measurement of unbalanced magnetic pull in cage induction motors with eccentric rotors. II. Experimental investigation, IEE Proceedings on Electric Power Applications, vol. 143, no. 3, pp. 202-210 (1996).

[7] Dorrell D.G., Chindurza I., Cossar C., Effects of rotor eccentricity on torque in switched reluctance Machines, IEEE Transactions on Magnetics, vol.41, no. 10, pp. 3961-3963 (2005).

[8] Sheth N.K., Rajagopal K.R., Effects of nonuniform airgap on the torque characteristics of a switched reluctance motor, IEEE Transactions on Magnetics, vol. 40, no. 4, pp. 2032-2034 (2004).

[9] Belahcen A., Arkkio A., Computation of additional losses due to rotor eccentricity in electrical machines, IEE Proceedings on Electric Power Applications, vol. 4, no. 4, pp. 259-266 (2009).

[10] Dorrell D.G., The influence of rotor eccentricity on the output torque of cage induction motors, International Conference on Electrical Machines, Paris, vol. 1, pp. 35-40 (1994).

[11] Faiz J., Ebrahimi B.M., Akin B., Toliyat H.A., Dynamic analysis of mixed eccentricity signatures at various operating points and scrutiny of related indices for induction motors, IET Proceedings on Electric Power Applications, vol. 4, no. 1, pp. 1-16 (2010).

[12] Faiz J., Ebrahimi B.M., Akin B., Toliyat H.A., Finite-Element Transient Analysis of Induction Motors Under Mixed Eccentricity Fault, IEEE Transactions on Magnetics, vol. 44, no. 1, pp. 66-74 (2008).

[13] Polat A., Ertuğrul Y.D., Ergene L.T., Static, dynamic and mixed eccentricity of induction motor, IEEE 10th International Symposium on Diagnostics for Electrical Machines, Power Electronics and Drives (SDEMPED), pp. 284-288 (2015).

[14] Sadowski N., Lefevre Y., Lajoie-Mazenc M., Cros, J., Finite element torque calculation in electrical machines while considering the movement, IEEE Transactions on Magnetics, vol. 28, no. 2, pp. 1410-1413 (1992).

[15] Marinescu M., Marinescu N., Numerical computation of torques in permanent magnet motors by Maxwell stresses and energy method, IEEE Transactions on Magnetics, vol. 24, no. 1, pp. 463-466 (1988).

[16] Reichert K., Freundl H., Vogt W., The calculation of forces and torques within numerical magnetic field calculation methods, Proceedings of COMPUMAG. Oxford, 31 March - 2 April 1976, Rutherford Laboratory, pp. 64-73 (1976).

[17] Coulomb J.L., A methodology for the determination of global electromechanical quantities from a finite element analysis and its application to the evaluation of magnetic forces, torques and stiffness, IEEE Transactions on Magnetics, vol. 19, no. 6, pp. 2514-2519 (1983).

[18] Silwal B., Rasilo P., Perkkiö L., Hannukainen A., Eirola T., Arkkio A., Numerical analysis of the power balance of an electrical machine with rotor eccentricity, IEEE Transactions on Magnetics, vol. 52, no. 3, pp. 1-4 (2016).

[19] Silwal B., Rasilo P., Perkkiö L., Oksman M., Hannukainen A., Eirola T., Arkkio A., Computation of torque of an electrical machine with different types of finite element mesh in the air gap, IEEE Transactions on Magnetics, vol. 1, no. 6, pp. 1-9 (2014).

[20] Arkkio A., Analysis of induction motors based on the numerical solution of the magnetic field and circuit equations, Acta Polytechnica Scandinavica (1987).

[21] Früchtenicht J., Jordan H., Seinsch H.O., Exzentnzitätsfelder als ursache von laufinstablilitäten bei asynchron-machinen. Parts 1 and 2, Archiv für Electrotechnik (in German), vol. 65, pp. 271-292 (1982).

[22] Nandi S., Bharadwaj R.M., Toliyat H.A., Parlos A.G., Performance analysis of a three phase induction motor under mixed eccentricity condition, IEEE Transactions on Energy Conversion, vol. 17, no. 3, pp. 392-399 (2002).

[23] Silwal B., Rasilo P., Haavisto A., Belahcen A., Arkkio A., Measurement of torque harmonics of a cage induction machine under rotor eccentricity, In Proceedings of International Conference on Electrical Machines and Systems, Pattaya, pp. 98-102 (2015). 